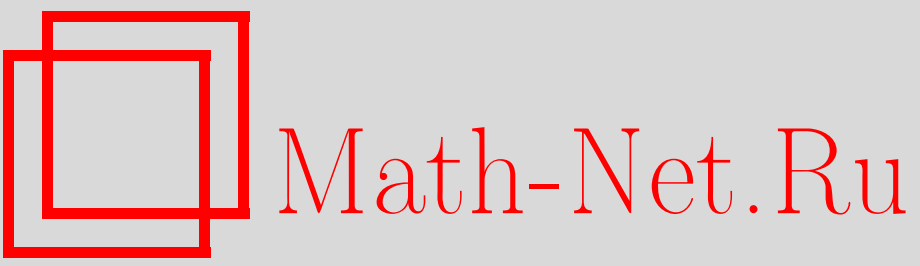

В. Д. Соловьев, Программные алгебры рекурсивных функций, УМН, 1996, том 51, выпуск 4, 125-144

DOI: https://doi.org/10.4213/rm996

Использование Общероссийского математического портала Math-Net.Ru подразумевает, что вы прочитали и согласны с пользовательским соглашением

http://www.mathnet.ru/rus/agreement

Параметры загрузки:

IP: 3.80 .253 .173

26 апреля 2023 г., 16:49:59 


\title{
ПРОГРАММНЫЕ АЛГЕБРЫ РЕКУРСИВНЫХ ФУНКЦИЙ
}

\author{
В. Д. СоловьЕв
}

\section{СОДЕРЖАНИЕ}

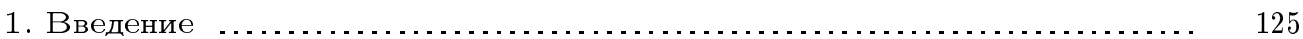

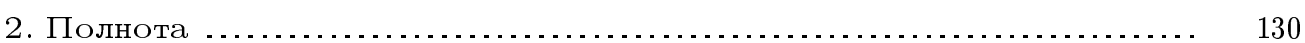

3. Максимальные подалгебры . . . . . . . . . . . . . . . . . . . . . . . . . . . 133

4. Модификации проблемы полноты ................................. 134

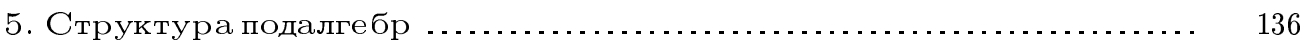

6. Конгруэнция и автоморфизмы ....................................... 139

7. Абстрактная вычислимость в алгебраических системах ............ 140

Список литературы ................................................ 142

\section{1. Введение}

В статье дается краткий обзор результатов по программным алгебрам рекурсивных функций. Под программной алгеброй понимается множество рекурсивных функций и предикатов вместе с некоторым классом схем программ в качестве операторов замыкания, действующих на этом множестве.

Алгебры рекурсивных функций можно трактовать так же, как функциональные системы в смысле [16]. Функциональная система это пара $\langle\mathscr{F}, \mathfrak{R}\rangle$, где $\mathscr{F}$ - множество функций, $\mathfrak{R}$ - множество операторов замькания на $\mathscr{F}$. К наиболее изученным функциональным системам относятся: двузначная логика [42], $k$-значная логика [31], автоматные функции [16].

Основные вопросы, изучаемые для функциональных систем, - проблема полноты; проблема выразимости; описание замкнутых классов (подалгебр), в первую очередь предполных (в алгебраической терминологии - максимальных подалгебр); описание автоморфизмов и конгруэнций.

Можно выделить три типа функциональных систем рекурсивных функций:

- только с операцией суперпозиции в качестве средств замыкания [17],

Работа выполнена при финансовой поддержке Российского фонда фундаментальных исследований (грант № 93-011-16004). 
- алгебры Робинсон [14] с алгебраическими средствами замыкания такими, как,+-1 ,

- с программными средствами замыкания.

В качестве программных средств замыкания рассматривались различные классы схем программ: стандартные, со счетчиками, с массивами и ряд других. Исследования программных алгебр рекурсивных функций естественно продолжают работы по алгебрам Робинсон, которые величь в 50-70 годах.

В этих исследованиях используется широкий спектр методов математической логики, теории алгоритмов, алгебры и математической кибернетики. Представляется интересным, что для описания подалгебр программных алгебр рекурсивных функций адекватным оказался язык теории категорий, а их свойства зависят от некоторых тонких результатов по группам Бернсайда. Изучение программных алгебр рекурсивных функций представляет интерес для обобщенной теории вычислимости, позволяя решить ряд проблем вычислимости в алгебраических системах, в частности, в полях.

Приведем основные определения.

ОПРЕДЕЛЕНИЕ 1. СХема программ с массивами и равенством-это конечньй ориентированный граф, все вершины которого помечены инструкциями. Инструкции могут иметь следуюший вид:
a) старт $\left(x_{1}, \ldots, x_{n}\right)$,
б) $y:=f\left(x_{1}, \ldots, x_{m}\right)$,
в) $y:=x$,
г) $x=y$,
д) $p\left(x_{1}, \ldots, x_{m}\right)$
e) $i:=i+1$,
ж) $i:=0$,
3) $i=j$,
и) $M[i]:=x$,
к) $x:=M[i]$,
л) стоп $(x)$,
м) стоп (TRUE),
н) стоп (FALSE).

Если вершина помечена инструкцией вида а), то в эту вершину никакая дуга не входит, и из нее ровно одна выходит; если инструкцией вида л), м), н), то в нее может входить несколько дуг, но из нее ни одна не выходит; если инструкцией вида г), д), з), то в такую вершину может входить несколько дуг, а выходят из нее ровно две дуги, причем одна помечена меткой TRUE, а другая меткой FALSE. Наконец, во всех остальных случаях входящих дуг может быть несколько, а выходящих ровно одна. Вершина с инструкцией вида а) должна быть единственной; в графе должна быть хотя бы одна вершина с инструкцией вида л), м) или н), причем, если присутствует вершина с инструкцией л), то не может быть вершин с инструкциями м), н).

Все переменные в инструкциях разделяются на два класса: предметные - $x, y, \ldots$, и счетчики $-i, j, \ldots$ И те, и другие принимают значения из множества натуральных 
чисел $\mathbb{N}$, но играют разную роль в схемах программ и не могут обмениваться значениями.

Буква $M$ обозначает бесконечный массив, состоящий из элементов $M[0], M[1], \ldots$; $f, p$ - функциональньй и предикатный символы, соответственно. Предметные переменные $x_{1}, \ldots, x_{n}$ в инструкции старт $\left(x_{1}, \ldots, x_{n}\right)$ являются входными, а переменная $x$ в инструкции стоп $(x)$ - выходной.

Определение 1 является стандартным в теории схем программ [15], [38] с той разницей, что введены операторы стоп (TRUE) и стоп (FALSE), позволяющие непосредственным образом определить вычислимость предикатов, а не только функций, и что разрешается использование предиката равенства для предметных переменных (инструкция г)).

Класс схем программ из определения 1 обозначим FDA= (аналогично [39]).

При подстановке в схему программ вместо функциональных и предикатных символов каких-либо конкретных рекурсивных функций и предикатов (скажем, из множества $\mathscr{L}$, получается интерпретированная (в $\mathscr{L}$ ) схема программ - конкретная программa.

Опишем семантику, т.е. способ выполнения программы.

ОПРЕДЕЛЕНИЕ 2. Полной памятью программи $\mathrm{ST}=\mathrm{ST}_{r} \cup \mathrm{ST}_{c}$ будем называть множество всех ее предметных переменных вместе со всеми ячейками массивов $\left(\mathrm{ST}_{r}\right)$ и множество всех ее счетчиков $\left(\mathrm{ST}_{r}\right)$. Оценкой назовем функцию $\mathrm{Val}=\mathrm{Val}_{r} \cup \mathrm{Val}_{c}$, где $\mathrm{Val}_{r}: \mathrm{ST}_{r} \rightarrow \mathbb{N}_{\text {и }} \mathrm{Val}_{c}: \mathrm{ST}_{c} \rightarrow \mathbb{N}$. Перед началом вьполнения программы функция Val нигде не определена.

Состоянием выполнения программы назьвается пара $u=(l, \mathrm{Val})$, где $l$-вершина граффа, Val - оценка. Выполнение программы описывается конечной или бесконечной последовательностью состояний $u_{0}, u_{1}, \ldots, u_{i}, u_{i+1}, \ldots$

Пусть $u_{i}=\left(l_{i}, \mathrm{Val}_{i}\right)$ и Инс-инструкция программы в вершине $l_{i}$.

а) Если Инс имеет вид старт $\left(x_{1}, \ldots, x_{n}\right)$ (это имеет место при $i=0$ ) и выходящая из вершины $l_{i}$ дуга ведет к вершине $k$, то $u_{i+1}=\left(k, \mathrm{Val}_{i+1}\right)$, причем $\mathrm{Val}_{i+1}$ отличается от $\mathrm{Val}_{i}\left(=\mathrm{Val}_{0}\right.$ - нигде не определенная функция) только на переменных $x_{1}, \ldots, x_{n}$ и $\forall j \leqslant n\left(\operatorname{Val}_{i+1}\left(x_{j}\right)=\right.$ вводимое значение переменной $\left.x_{j}\right)$.

б) Если Инс имеет вид стоп $(x)$, стоп(TRUE) или стоп(FALSE), то последовательность состояний конечна - программа останавливается с результатом $\operatorname{Val}_{i}(x)$, TRUE или FALSE, соответственно.

в) Если Инс имеет вид $x:=f_{j}\left(x_{1}, \ldots, x_{m}\right)$ или $x:=y$, а выходяшая из вершины $l_{i}$ дуга ведет к вершине $k$, то $u_{i+1}=\left(k, \mathrm{Val}_{i+1}\right)$, причем $\mathrm{Val}_{i+1}$ отличается от $\mathrm{Val}_{i}$

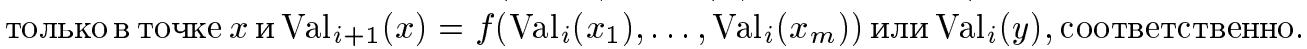

г) Пусть Инс имеет вид $p\left(x_{1}, \ldots, x_{m}\right)$ или $x=y$. Если $p\left(\operatorname{Val}_{i}\left(x_{1}\right), \ldots, \operatorname{Val}_{i}\left(x_{m}\right)=\right.$ TRUE или $\operatorname{Val}_{i}(x)=\operatorname{Val}_{i}(y)$, соответственно, и выходящая из $l_{i}$ дуга с меткой TRUE ведет к вершине $k_{1}$, то $u_{i+1}=\left(k_{1}, \mathrm{Val}_{i+1}\right)$, где $\mathrm{Val}_{i+1}=\mathrm{Val}_{i}$. Если же

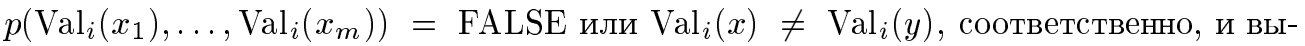
ходящая из $l_{i}$ дуга с меткой FALSE ведет к вершине $k_{2}$, то $u_{i+1}=\left(k_{2}, \mathrm{Val}_{i+1}\right)$, где $\mathrm{Val}_{i+1}=\mathrm{Val}_{i}$.

д) Если Инс имеет вид $M[j]:=x$ и выходящая из $l_{i}$ дуга ведет к вершине $k$, то 
$u_{i+1}=\left(k, \operatorname{Val}_{i+1}\right)$, причем $\operatorname{Val}_{i+1}\left(M\left[\operatorname{Val}_{i}(j)\right]\right)=\operatorname{Val}_{i}(x)$, а в остальных точках $\operatorname{Val}_{i+1}$ совпадает с $\operatorname{Val}_{i}$.

е) Если Инс имеет вид $x:=M[j]$ и выходяшая из $l_{i}$ дуга ведет к вершине $k$, то $u_{i+1}=\left(k, \operatorname{Val}_{i+1}\right)$, причем $\operatorname{Val}_{i+1}(x)=\operatorname{Val}_{i}\left(M\left[\operatorname{Val}_{i}(j)\right]\right)$, а в остальных точках $\operatorname{Val}_{i+1}$ совпадает с $\mathrm{Val}_{i}$.

ж) Если Инс имеет вид $j:=j+1$ или $j:=0$ и выходяшая из $l_{i}$ дуга ведет к вершине $k$, то $u_{i+1}=\left(k, \operatorname{Val}_{i+1}\right)$, причем $\operatorname{Val}_{i+1}(j)=\operatorname{Val}_{i}(j)+1$ и $\operatorname{Val}_{i+1}(j)=0$, соответственно, а в остальных точках $\mathrm{Val}_{i+1}$ совпадает с $\mathrm{Val}_{i}$.

з) Пусть Инс имеет вид $j_{1}=j_{2}$. Если $\operatorname{Val}_{i}\left(j_{1}\right)=\operatorname{Val}_{i}\left(j_{2}\right)$ и выходящая из $l_{i}$ дуга с меткой TRUE ведет к вершине $k_{1}$, то $u_{i+1}=\left(k_{1}, \operatorname{Val}_{i+1}\right)$, причем, $\operatorname{Val}_{i+1}=\operatorname{Val}_{i}$. Если же $\operatorname{Val}_{i}\left(j_{1}\right) \neq \operatorname{Val}_{i}\left(j_{2}\right)$ и выходящая из $l_{i}$ дуга с меткой FALSE ведет к вершине $k_{2}$, то $u_{i+1}=\left(k_{2}, \mathrm{Val}_{i+1}\right)$, причем $\mathrm{Val}_{i+1}=\mathrm{Val}_{i}$.

Если при вычислении $\mathrm{Val}_{i+1}$ используемое значение $\mathrm{Val}_{i}$ неопределено, то $\mathrm{Val}_{i+1}$ тоже неопределено. В пункте в), если функция $f$ неопределена, то $\mathrm{Val}_{i+1}(x)$ неопределено. В пункте г), если предикат $p$ неопределен или равенство $\operatorname{Val}_{i}(x)=\operatorname{Val}_{i}(y)$ принимает неопределенное значение в силу неопределенности значений переменных $x$ и $y$, то вьполнение программы на этом прерьвается (последовательность состояний конечна) с неопределенньм результатом.

Перечислим ряд определений классов схем программ, получаюшихся из определения 1 простыми изменениями.

Если в определении 1 опустить инструкции вида и) и к), то получится определение схем программ со счетчиками - FDC.

Если, кроме того, убрать и инструкции е), ж), з), то получится определение стандартных схем программ $-\mathrm{FD}_{=}$.

Если во всех перечисленных определениях опустить инструкцию г), то соответствуюшие классы обозначаются FDA, FDC, FD.

Если в определении $\mathrm{FD}\left(\mathrm{FD}_{=}\right)$добавить инструкции $\operatorname{puch}(x), x:=$ рор для работы с магазином (одним), то получаюшийся класс обозначается FDS (FDS=).

Если в определении FD (FDS) допускается использование лишь одной единственной предметной переменной $x$, то получающийся класс обозначается $\operatorname{FD}(1)(\operatorname{FDS}(1))$.

Если к FDA = добавить инструкцию $x:=M[y]$, т.е. разрешить использовать предметные переменные в качестве счетчиков, то получается класс схем программ с косвенной адресацией, который будет обозначаться FDAA=.

Kласс FDS, как известно [15], эквивалентен рекурсивным программам. $\mathrm{FD}(1)-$ это класс схем Янова [32]. Класс $\operatorname{FDS}(1)$, изучавшийся в [47], эквивалентен системам алгоритмических алгебр Глушкова [2]. Класс FDAA = по существу есть класс РАМ-машин [33].

Для любого множества рекурсивных функций и предикатов $D$ каждый из классов схем программ определяет совокупность операторов замыкания на $D$ следуюшим образом.

ОПРЕДЕЛЕНИЕ 3 . Пусть $S$-произвольная схема программ из класса $K$ и $\mathscr{L}$ - упорядоченный набор рекурсивных функций и предикатов, элементы которого определенным образом подставляются вместо функциональных и предикатных символов в $S$. Если при этом вычисляется некоторая функция $f$ (или предикат $p$ ) из $D$, то говорят, 
что схема $S$ преобразует набор $\mathscr{L}$ в $f($ в $p$ ) и тем самым определяет оператор замыкания на $D$.

Обозначим через $[\mathfrak{N}]_{K}^{D}$ замыкание в $D$ множества $\mathfrak{N}$ рекурсивных функций и предикатов относительно класса схем программ $K$, т.е. множество всех функций и предикатов из $D$, вычислимых схемами программ из $K$, интерпретированными в $\mathfrak{N}$. Если понятно о каких $D$ и $K$ идет речь, то они могут опускаться.

Пара $\langle D, K\rangle$ будет называться программной алгеброй рекурсивных функций и предикатов с множеством схем программ $K$ в качестве операторов замыкания.

Если класс $K$ схем программ фиксирован или несущественно, о каких $K$ идет речь, и не может возникнуть недоразумений, то упоминание о нем будет опускаться, а алгеброй будет называться также и просто множество функций и предикатов (замкнутое относительно $K)$.

Подмножество $\mathfrak{N}$ множества $D$ называется подалгебр й, если оно замкнуто относительно $K$, т.е. $\mathfrak{N}=[\mathfrak{N}]_{K}^{D}$. Подалгебра является конечно-порожденной, если $\mathfrak{N}=[\mathscr{L}]_{K}^{D}$ для некоторого конечного $\mathscr{L}$. Наконец, множество $\mathfrak{N}$ называется системой образующих или полныцм (в $D)$, если $[\mathfrak{N}]_{K}^{D}=D$.

Одной из основных трудностей при изучении программных алгебр рекурсивных функций является многочисленность алгебр с сушественно различными свойствами и требующих различных методов для их изучения. В работах по программным алгебрам основное внимание было уделено алгебре одноместных общерекурсивных функций с операторами замькания FDA= и алгебре одноместных частично рекурсивных функций с операторами замыкания $\operatorname{FDS}(1)$, которые можно рассматривать как модельные.

Далее будут использоваться следующие обозначения: $o(x)=0, s(x)=x+1$, $s^{-}(x)=x-1, \operatorname{id}(x)=x$ (для функций), $P_{0}(x) \Leftrightarrow x=0$ (для предиката) и $\mathbb{N}$ - для множества натуральных чисел, включая 0. Запись $f(x)$ ! для частичной функции $f$ означает, что $f$ в точке $x$ определена.

$\langle A, \mathscr{L}\rangle$ обозначает алгебраическую систему с основным множеством $A$ и сигнатурой $\mathscr{L}=\left\langle f_{1}, \ldots, f_{n}, p_{1}, \ldots, p_{m}\right\rangle$, состоящей из функций $f_{1}, \ldots, f_{n}$ и предикатов $p_{1}, \ldots, p_{m}$

Множество одноместных частично (обще-) рекурсивных функций и предикатов будем обозначать $\mathscr{F}_{r}^{1}\left(\mathscr{F}_{0}^{1}\right)$, многоместных - $\mathscr{F}_{r}\left(\mathscr{F}_{0}\right)$, соответственно. Аналогичны обозначения для множеств предикатов: $\mathscr{P}_{r}^{1}, \mathscr{P}_{0}^{1}, \mathscr{P}_{r}, \mathscr{P}_{0}$.

Следующие обозначения соответствуют [21]:

$\pi(x, y)$ - общерекурсивная функция, нумерующая пары натуральных чисел,

$D_{0}, D_{1}, \ldots$ - эффективная нумерация конечных множеств,

$W_{0}, W_{1}, \ldots$ - эффективная нумерация рекурсивно перечислимых множеств,

$\varphi_{0}, \varphi_{1}, \ldots$ - эффективная нумерация частично рекурсивных функций,

$\psi_{0}, \psi_{1}, \ldots$ - эффективная нумерация частично рекурсивных предикатов.

Необходимые для понимания статьи определения и результаты по теории рекурсивных функций могут быть найдены в [21]. 


\section{2. Полнота}

Первыми работами по программным алгебрам рекурсивных функций можно считать работы [10], [46], где были приведены первые простые примеры полных наборов такие, как $\{o, s,=\}$ и $\left\{s, s^{-}, P_{0}\right\}$. Указанные наборы являются полными в множестве всех частично рекурсивных функций и предикатов при использовании в качестве средств замыкания класса FD стандартных схем программ или более сильных программных формализмов.

Иным оказывается положение дел в алгебрах с классами $\mathrm{FD}(1)$ и $\mathrm{FDS}(1)$ в качестве средств замыкания. Указанные выше наборы уже не будут там полными. Пример полного набора для $\mathrm{FD}(1)$ (и для $\operatorname{FDS}(1)$ ) предложен М. Минским [18] - это $\left\{2 x, 3 x, 2^{x},[x / 6],\left[\log _{2} x\right], x=0(\bmod 6)\right\}$. Из одного результата Я. Барздиня [1] следует, что без операций $2^{x}$ и $\left[\log _{2} x\right]$ свойство полноты теряется.

В [12] поставлена проблема полноты как проблема нахождения общего критерия полноты произвольной системы рекурсивных функций и предикатов, без уточнения средств замыкания, для которых эта проблема должна быть решена. Такой критерий не может быть эффективным - в [13] для графо-схем с памятью (по сушеству эквивалентных стандартным схемам программ) показано, что проблема полноты алгоритмически неразрешима. В [26] этот результат уточнен определением степени алгоритмической неразрешимости (T-степени, [21]) проблемы полноты.

ОПРЕДЕЛЕНИЕ 4. Множество

$$
\begin{array}{r}
I_{\langle D, K\rangle}=\left\{\pi(x, y) \mid \exists i_{1}, \ldots, i_{n}, j_{1}, \ldots, j_{m}\left(D_{x}=\left\{i_{1}, \ldots, i_{n}\right\} \& D_{y}=\left\{j_{1}, \ldots, j_{m}\right\}\right.\right. \\
\left.\left.\& \mathscr{L}=\left\langle\varphi_{i_{1}}, \ldots, \varphi_{i_{n}}, \psi_{j_{1}}, \ldots \psi_{j_{m}}\right\rangle \text { полон в алгебре }\langle D, K\rangle \& \mathscr{L} \subseteq D\right)\right\}
\end{array}
$$

назьвается индексным множеством проблемы полноты алгебры $\langle D, K\rangle$.

Tеорема 1. T-степень множества $I_{\left\langle\mathscr{F}_{0}, \mathrm{FDA}_{=}\right\rangle}$есть $0^{\prime \prime}$.

ТЕОРема 2. Множество $I_{\left\langle\mathscr{F}_{0}, \mathrm{FDA}_{=}\right\rangle}$принадлежит уровню $\left(\Sigma_{3} \cap \Pi_{3}\right) \backslash\left(\Sigma_{2} \cup \Pi_{2}\right)$ иерархии Клини-Мостовского.

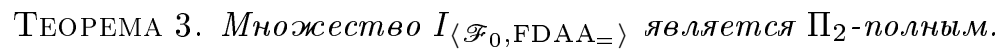

TEOPEмA 4. [8]. T-степень множсества $\left.I_{\langle\mathscr{F} 1,}, \operatorname{FDS}(1)\right\rangle$ есть $0^{\prime \prime \prime}$.

В работе [8] также определены степени неразрешимости проблемы полноты для ряда алгебр Робинсон - для разных алгебр это степени $0^{\prime \prime}, 0^{\prime \prime \prime} 0^{\text {iv }}$ или $0^{\text {iv }}$. В [19] В. Непомняший сформулировал программу сравнения различных классов схем программ по сложности решения проблемы полноты для соответствуюших алгебр. Результаты и методы, развитые в [8], [26], дают одно из возможных решений поставленной задачи.

После установления отсутствия эффективного критерия, поиск решения проблемы полноты был предложен в трех направлениях: 1) нахождение критерия полноты, сформулированного на логическом языке в терминах функциональных свойств функций и предикатов, входящих в заданную систему, 2) описание всех максимальных подалгебр (что позволяет описать полные системы, по аналогии с постовским критерием полноты для двузначных логик, как не содержашиеся ни в одной максимальной подалгебре), 3) модификация проблемы полноты. 
В рамках первого направления [19] В. Непомняшим изучалась алгебра с операторами замыкания из класса $\mathrm{FD}_{=}$и дополнительной операцией $x:=0$. Для наборов, состоящих из одной функции, получен критерий полноты следуюшего вида:

ТеОрема 5. Набор $\{f\}$ полон тогда и только тогда, когда

$$
\left\{0, f(0), \ldots, f^{n}(0), \ldots\right\}=\mathbb{N} .
$$

Следуюшим шагом в этом направлении явился критерий полноты произвольной конечной системы для алгебры $\left\langle\mathscr{F}_{0}, \mathrm{FDA}_{=}\right\rangle$[23]. Его можно рассматривать как обобшение критерия В. Непомнящего.

ТЕОРема 6 (критерий полноты для $\left.\left\langle\mathscr{F}_{0}, \mathrm{FDA}_{=}\right\rangle\right)$). Набор общерекурсивных функиий и предикатов $\mathscr{L}=\left\langle f_{1}, \ldots, f_{n}, p_{1}, \ldots, p_{m}\right\rangle$ полон тогда и только тогда, когда

1) $\forall x, y \exists \tau(\tau-$ терм сигнатуры $\mathscr{L} \& \tau(x)=y)$,

2) $\exists a \exists \Phi(\Phi(x)$ - бескванторная формула сигнатуры $\mathscr{L} \cup\{=\} \& \Phi(a)$ $\& \forall y \neq a\rceil \Phi(y))$.

Сформулированный критерий содержит двухкванторные условия. Из упомянутого вьшше результата о том, что $T$-степень проблемы полноты для алгебры $\left\langle\mathscr{F}_{0}, \mathrm{FDA}=\right\rangle$ есть $0^{\prime \prime}$, можно сделать вывод, что критерий полнотыне может состоять лишь из однокванторных условий (иначе $T$-степень проблемы полноты была бы $0^{\prime}$ ), т.е. что предложенньй критерий полноты является практически простейшим возможным.

В [26], [28] сформулированный критерий перенесен (с применением результатов работы [38]) на другие классы схем программ, причем для класса FDS= критерий полноты совпадает с критерием полноты для $\mathrm{FDA}=$.

Tеорема $7\left(\left\langle\mathscr{F}_{0}, \mathrm{FDAA}_{=}\right\rangle\right)$. Набор $\mathscr{L}$ полон тогда и только тогда, когда $\forall x, y \exists \tau(\tau-$ терм сигнатуры $\mathscr{L} \& \tau(x)=y)$.

Наиболее сложным является критерий полноты для алгебры $\left\langle\mathscr{F}_{0}, \mathrm{FDC}_{=}\right\rangle$.

Скажем, что переменная $x$ вычисляется в сигнатуре $\mathscr{L}$ исходя из набора $\left(a_{1}, \ldots, a_{k}\right)$ с использованием не более $l$ переменных, если существует конечная последовательность присваиваний вида $z:=f\left(y_{1}, \ldots, y_{n}\right)$ такая, что

а) функции, используемые в присваиваниях, входят в сигнатуру $\mathscr{L}$,

б) в последнем присваивании $x$ стоит слева от знака :=,

в) во всех присваиваниях последовательности вместе взятых используется не более $l$ переменных,

г) в правой части любого присваивания могут использоваться лишш переменные $a_{1}, \ldots a_{k}$ и переменные, встретившиеся в левых частях предшествуюших присваиваний.

TeOpema 8. $\left(\left\langle\mathscr{F}_{0}, \mathrm{FDC}_{=}\right\rangle\right)$. Набор $\mathscr{L}$ полон тогда и только тогда, когда

1) $\forall x, y \exists \tau(\tau-$ терм сигнатуры $\mathscr{L} \& \tau(x)=y)$,

2) $\exists a \exists \Phi(\Phi(x)$ - бескванторная формула сигнатурь $\mathscr{L} \cup\{=\} \& \Phi(a) \&$ $\forall y \neq a\rceil \Phi(y))$, 
3) $\forall k \exists l \forall a_{1}, \ldots, a_{k} \forall x \forall \tau\left(\tau-\right.$ терм сигнатуры $\mathscr{L} \& \tau\left(a_{1}, \ldots, a_{k}\right)=x \rightarrow x$ может бить вычислен в сигнатуре $\mathscr{L}$ исходя из набора $\left(a_{1}, \ldots, a_{k}\right)$ с использованием не более $l$ переменных).

До сих пор не найдены аналогичные критерии полноты для алгебр с операторами замыкания $\mathrm{FD}(1)$ и $\operatorname{FDS}(1)$.

Приведем простые примеры систем полных в одной алгебре, но не полных в другой.

ПримеР 1 [37]. Система $\{o, g\}$, где

$$
g(i, j)= \begin{cases}j+1, & \text { если } i=[j / 2], \\ 0 & \text { в противном случае, }\end{cases}
$$

полна в $\left\langle\mathscr{F}_{r}, \mathrm{FDA}_{=}\right\rangle$, но не полна в $\left\langle\mathscr{F}_{r}, \mathrm{FDC}_{=}\right\rangle$.

ПримеР 2 [7]. Система $\left\{s, s^{-}, P\right\}$ полна в $\left\langle\mathscr{F}_{r}, \mathrm{FD}\right\rangle$, но не полна в $\left\langle\mathscr{F}_{r}, \operatorname{FDS}(1)\right\rangle$.

Сопоставление полученных критериев полноты позволяет сравнить классы схем программ (т.е. фактически различные концепции алгоритмов) в духе программы В. Непомнящего [19].

Сравнивая формулировки теорем $5,6,7$ и упомянутьй результат для алгебры $\left\langle\mathscr{F}_{0}, \mathrm{FDS}_{=}\right\rangle$, можно заметить обшую закономерность: чем сильнее средства замыкания, тем проше решается проблема полноты. В пользу этого вывода свидетельствует и то, что для слабых средств замыкания $\mathrm{FD}(1)$ и $\mathrm{FDS}(1)$, как отмечено выше, критерия полноты получить не удалось.

Еще одним аргументом является сравнение степеней неразрешимости индексных

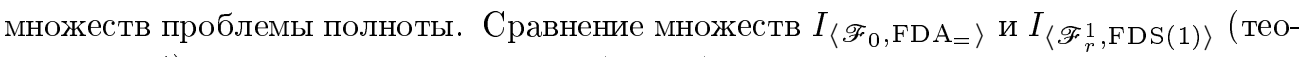
ремы 1 и 4) показывает, что для алгебры с более сильными средствами замыкания степень неразрешимости индексного множества проблемы полноты меншше, т.е. оно алгоритмически проще. Сопоставление теорем 2 и 3 показывает, что для множеств $I_{\left\langle\mathscr{F}_{0}, \mathrm{FDA}_{=}\right\rangle}$и $I_{\left\langle\mathscr{F}_{0}, \mathrm{FDAA}_{=}\right\rangle}$, хотя их $T$-степени и равны $\left(=0^{\prime \prime}\right)$, но их $m$-степени различаются - для алгебры с более сильными средствами замыкания соответствуюшая $m$-степень строго меньше.

В тех случаях, когда для какого-либо класса функций некоторая проблема оказывается алгоритмически неразрешимой, обычно стремятся выделить содержательные подклассы этого класса функций, для которых данная проблема окажется уже разрешимой.

Сформулированные вьше критерии полноты позволяют показать, что в классе многочленов с целыми коэффициентами проблема полноты разрешима. Так как все вычисления выполняются в множестве $\mathbb{N}$, то отрицательные значения многочленов будем заменять на число 0. Назовем получающиеся в результате функции многочленами на $\mathbb{N}$.

ТЕОрема 9. Существует алгоритм, который по любому конечному множеству многочленов на $\mathbb{N}$ определяет, является ли оно полным в алгебре $\left\langle\mathscr{F}_{0}, \mathrm{FDA}_{=}\right\rangle$. 


\section{3. Максимальные подалгебры}

Изучение максимальных подалгебр не только позволяет продвинуться в решении вопроса о полноте, но и позволяет получить информацию о строении подалгебр вообще.

Максимальные подалгебры изучались в [14] для алгебр Робинсон рекурсивных функций, но не были полностью описаны.

Для алгебры $\left\langle\mathscr{F}_{0}^{1}, \mathrm{FDA}_{=}\right\rangle$сформулированньй вьше критерий полноты позволяет построить следующую классификацию максимальных подалгебр.

K1.1. Совокупность всех максимальных подалгебр $\mathfrak{A}$ таких, что

а) $\exists a \exists \Phi(\Phi(x)$ - бескванторная формула сигнатуры $\mathfrak{A} \cup\{=\} \& \Phi(a) \& \forall y \neq$ $a\rceil \Phi(y))$,

б) $\exists x, y \forall g \in \mathfrak{A}(y \neq g(x))$.

K1.2. Совокупность всех максимальных подалгебр $\mathfrak{A}$ таких, что

а) $\exists a \exists \Phi(\Phi(x)$ - бескванторная формула сигнатуры $\mathfrak{A} \cup\{=\} \& \Phi(a) \& \forall y \neq$ $a\rceil \Phi(y))$,

б) $\forall x, y \exists g \in \mathfrak{A}(y=g(x))$,

в) $\rceil \exists \mathscr{L} \subseteq \mathfrak{A}(\mathscr{L}$ конечно $\& \forall x, y \exists \tau(\tau-$ терм сигнатуры $\mathscr{L} \& y=\tau(x)))$.

К2.1. Совокупность всех максимальных подалгебр $\mathfrak{A}$ таких, что

а) $\exists D \exists \Phi(D$ - конечное множество \& $\Phi(x)$ - бескванторная формула сигнатурьи $\mathfrak{A} \cup\{=\} \& \forall a((a \in D \rightarrow \Phi(a)) \&(a \notin D \rightarrow\rceil \Phi(a))))$,

б) $\rceil(\exists a \exists \Phi(\Phi(x)$ - бескванторная формула сигнатуры $\mathfrak{A} \cup\{=\} \& \Phi(a) \& \forall y \neq$ $a\rceil \Phi(y)))$,

в) $\exists \mathscr{L} \subseteq \mathfrak{A}(\mathscr{L}$ конечно $\& \forall x, y \exists \tau(\tau-$ терм сигнатуры $\mathscr{L} \& y=\tau(x)))$.

К2.2. Совокупность всех максимальных подалгебр $\mathfrak{A}$ таких, что

а) $\exists \mathscr{L} \subseteq \mathfrak{A}(\mathscr{L}$ конечно \& $\forall x, y \exists \tau(\tau-$ терм сигнатурь $\mathscr{L} \& y=\tau(x)))$,

б) $\rceil \exists D \exists \Phi(D-$ конечное множество \& $\Phi(x)$ - бескванторная формула сигнатурь $\mathfrak{A} \cup\{=\} \& \forall a((a \in D \rightarrow \Phi(a)) \&(a \notin D \rightarrow\rceil \Phi(a))))$.

Описание этих совокупностей дано в работах [24], [25].

ТЕОРема 10. Все совокупности кроме К2.1 континуальны.

В алгебре $\left\langle\mathscr{F}{ }_{r}^{1}, \operatorname{FDS}(1)\right\rangle$ описано несколько совокпностей максимальных подалгебр (см. [4]-[6], [22]), однако это описание не является исчерпьвающим.

В качестве примера приведем три совокупности максимальных подалгебр (алгебры $\left.\left\langle\mathscr{F}_{r}^{1}, \operatorname{FDS}(1)\right\rangle\right)$ интересных тем, что в их описании используются понятия классической теории алгоритмов.

Теорема 11 [4]. Для множества $A \subseteq \mathbb{N}$ подалгебра $\mathfrak{A}_{A}=\{f \mid f(A) \cap \bar{A}=\varnothing\}$ $\cup \mathscr{P}_{r}^{1}$ является максимальной тогда и только тогда, когда А конечно или коконечно.

Алгебра $\mathfrak{A}_{A}$ называется алгеброй сохранения принадлежсности множеству. 
ОПРедЕЛЕниЕ 5 [6]. Сжатое множество $F$ называется наибольиим, если $\forall W$ ( $W$ рекурсивно перечислимо \& $F \nsubseteq W \rightarrow \exists R(R-$ рекурсивное множество \& $F \varsubsetneqq$ $R \varsubsetneqq W)$ ).

Tеорема 12 [6]. Если множество $A \subseteq \mathbb{N}$ является

а) бесконечным рекурсивным с бесконечным дополнением или

б) наибольшим сжатылм множеством, то подалгебра $\mathfrak{A}_{A}^{\prime}=\{f \mid f(\bar{A}) \cap A$ конечно $\cup \cup \mathscr{P}_{r}^{1}$ максимальна.

ОПРЕДЕЛЕНИЕ 6 [6]. Сжатые множества $A$ и $B$ называются $f$-эквивалентными тогда и только тогда, когда существует частично-рекурсивная функция $\varphi$ такая, что $\varphi(A)$ и $B$ почти совпадают (т.е. отличаются лишш на конечное множество чисел).

Квазисжсатым [21] называется множество, являющееся конечным объединением сжатых множеств.

Tеорема 13 [6]. Если $A$ - квазисжатое множсество и $A=\bigcup_{i=1}^{k} A_{i}$, где $A_{i}-$ сжсатые $, i=1, \ldots, k$, то алгебра $\mathfrak{A}_{A}^{\prime}=\{f \mid f(\bar{A}) \cap A$ конечно $\} \cup \mathscr{P}_{r}^{1}$ является максимальной тогда и только тогда, когда все множества $A_{i}, i=1, \ldots, k,-$ наибольшие сжсатые и попарно $f$-эквивалентнье.

Сравнение полученных описаний максимальных подалгебр в программных алгебрах с максимальными подалгебрами в алгебрах Робинсон позволяет сделать вывод [5] об их несопоставимости. При этом программные алгебры имеют в целом более регулярное строение.

Также существенно отличаются максимальные подалгебры и в различных программных алгебрах рекурсивных функций. Например, в алгебре $\left\langle\mathscr{F}_{r}^{1}, \operatorname{FDS}(1)\right\rangle$ подалгебра Фраттини (пересечение всех максимальных подалгебр) не имеет конечных систем образующих [4], в то время как в алгебре $\left\langle\mathscr{F}_{0}^{1}, \mathrm{FDA}_{=}\right\rangle$подалгебра Фраттини является тривиальной - это наименьшая подалгебра $U_{0}=\{$ TRUE, FALSE, id $\}$.

В ряде работ программные алгебры рекурсивных функций и предикатов расшепляются на две компоненты $\langle F, R\rangle$, где $F$ - множество ее функций, $R$ - множество ее предикатов, и каждая из них изучается отдельно.

ОПРЕДЕЛЕНИЕ 7. Подалгебра $\langle F, R\rangle$ назьвается максимальной по функииям, если добавление к ней не содержашейся в ней функции с последующим замьканием приводит к алгебре всех рекурсивных функций и предикатов.

Аналогично определяется максимальность по предикатам. В [22] показано существенное различие между этими компонентами.

TЕОРема 14. Если подалгебра алгебры $\left\langle\mathscr{F}_{r}^{1}, \operatorname{FDS}(1)\right\rangle$ максимальна по функииям, то она максимальна и по предиктам, обратное же не верно: существуют подалгебры, максимальные по предикатам, но не максимальные по функииям.

\section{4. Модификации проблемы полноты}

Первоначально модифицированная проблема полноты рассматривалась для автоматных функций [16]. Для алгебры $\left\langle\mathscr{F}_{r}^{1}, \operatorname{FDS}(1)\right\rangle$ модифицированные проблемы полноты изучались в [7], [9]. 
ОПРЕДЕЛЕНИЕ 8. [7]. Множество функций и предикатов $M$ называется $A$-nолным, если

$$
\forall t \in \mathscr{F}_{r}^{1} \exists z \exists h \in[M]_{\mathrm{FDS}(1)} \forall x \leqslant z(t(x)=h(x)) .
$$

Множество $M$ назьвается $A$-предполным, если оно не $A$-полно, но становится таковьм после добавления к нему любого элемента из $\mathscr{F}_{r}^{1}-M$.

Обозначим $\Phi=\left\{\mathfrak{A}_{A} \mid A \neq \mathbb{N} \& A \neq \varnothing\right\}$ ( $\mathfrak{A}_{A}$ из теоремы 11). Далее, пусть $w-$ не диагональное бинарное симметричное и рефлексивное отношение на $\mathbb{N}$, и $\Psi$ - семейство подалгебр вида

$$
\begin{aligned}
& \mathfrak{A}(w)=\{f \mid \forall a, b((a, b) \in w \& f(a) ! \& f(b) !\rightarrow(f(a), f(b)) \in w)\} \\
& \cup\{p \mid \forall a, b((a, b) \in w \& p(a) ! \& p(b) ! \rightarrow p(a) \equiv p(b))\}
\end{aligned}
$$

для всех таких $w$. Как видно из следующего результата, $A$-предполные классы устроены проще, чем максимальные подалгебры.

Tеорема 15 [7]. Множество A-предполных классов есть $\Phi \cup \Psi$.

Несмотря на наличие этого достаточно простого описания, проблема $A$-полноты остается по-прежнему эффективно неразрешимой, а число $A$-предполных классов по-прежнему континуально.

ОПРЕДЕЛЕНИЕ 9. Множество подалгебр T называется $\alpha$-критериальной системой (для $A$-полноты), если для любого множества функций и предикатов $M$ мошности, меньшей чем $\alpha, M A$-полно тогда и только тогда, когда множество $M$ не содержится ни в одной из подалгебр множества $T$.

Известно [16], что сушествуют счетные $\aleph_{0}$-критериальные системы. В [7] впервые дано конструктивное описание счетной $\aleph_{0}$-критериальной системы.

ТЕОрема 16. Множсество $\left\{\mathfrak{A}_{A} \mid A\right.$ рекурсивно пречислимо, не просто $\} \cup$ $\{\mathfrak{A}(w) \mid w$ - рекурсивно перечислимо, не просто $\}$ является счетной $\aleph_{0}$-критериальной системой для А-полноть.

Другая модификация проблемы полноты рассматривалась в [9].

ОПРЕДЕЛЕНИЕ 10. Функция $t$ назьвается $\varphi$-аппроксимирующей функцией $f$, если в их области определения $\Delta t$ и $\Delta f$ совпадают и $\forall x \in \Delta f(|f(x)-t(x)| \leqslant \varphi(x))$. Множество $M$ функций и предикатов назьвается $\varphi$-полным, если оно содержит предикат с непустьми областями истинности и ложности, и для всякой функции $f \in \mathscr{F}_{r} 1$ замыкание $[M]_{\mathrm{FDS}(1)}$ содержит $\varphi$-аппроксимирующую ее функцию. Множество $M$ назьвается слабо полнылм, если оно $\varphi$-полно для некоторой общерекурсивной функции $\varphi$.

ТЕОРемА 17. m-степень индексного множсества проблемы полноты меньше или равна т-степени индексного множества проблемы бой общерекурсивной функиии $\varphi$, а также и проблемы слабой полноты.

Таким образом, эта модификация не упрощает решение проблемы полноты. 


\section{5. Структура подалгебр}

Следующим шагом в изучении программных алгебр является изучение структуры всех ее подалгебр. Ясно, однако, что в силу ее континуальности и крайней сложности даже максимальных подалгебр, рассчитьвать на полное описание этой структуры вряд ли возможно. Поэтому согласно [3] внимание в первую очередь должно быть сосредоточено на подалгебрах, достижимых сверху за конечное число шагов (так называемых подалгебрах конечной глубины), т.е. следует изучить совокупность таких подалгебр $\mathfrak{A}$, что $\exists n>1 \exists \mathfrak{A}_{1}, \ldots, \mathfrak{A}_{n}\left(\mathfrak{A}=\mathfrak{A}_{1} \& \forall i\left(1<i \leqslant n \rightarrow \mathfrak{A}_{i-1}\right.\right.$ является максимальной подалгеброй $\mathfrak{A}_{i} \& \mathfrak{A}_{i-1}$ является конечно порожденной).

ОпрЕДЕлЕниЕ 11. Группа $G$ перестановок множества $A$ назьвается полурегулярной, если

$$
\forall g \in G \forall a \in A(g(a)=a \rightarrow \forall x \in A(g(x)=x)) .
$$

ОПРЕДЕЛЕНИЕ 12. Отображение $f: A \rightarrow B$ назовем $p$-гомоморфизмом, если на множествах $A$ и $B$ определены полурегулярные групшы перестановок $G_{1}$ и $G_{2}$, соответственно, и

$\forall g_{1} \in G_{1} \forall g_{2} \in G_{2} \forall x \in A\left(f\left(g_{1}(x)\right)=g_{2}(f(x)) \rightarrow \forall y \in A\left(f\left(g_{1}(y)\right)=g_{2}(f(y))\right)\right)$

ОПРЕДЕЛЕНИЕ 13. Пару $p$-гомоморфизмов $f_{1}, f_{2}: A \rightarrow B$ назовем регулярной, если

$$
\forall x \in A\left(f_{1}(x)=f_{2}(x) \rightarrow \forall y \in A\left(f_{1}(y)=f_{2}(y)\right)\right) .
$$

ОПРЕДЕЛЕНИЕ 14. Сетью вложсений Е назовем четверку

$$
\left\langle\left\{A_{i}\right\}_{i \in I}, \leqslant D,\left\{G_{i}\right\}_{i \in I}, E\right\rangle
$$

где $I$ - конечное множество индексов: $\left\{A_{i}\right\}_{i \in I}$ - совокупность конечных множеств;

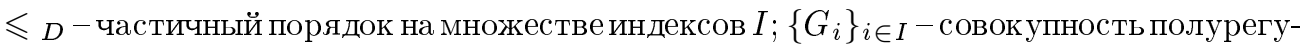
лярных групп перестановок на множествах $\left\{A_{i}\right\}_{i \in I}$ с соответствующими индексами; $E$ - совокупность множеств $\left\{\alpha_{i j}\right\}_{i, j \in I, i \leqslant{ }_{D} j}$-гомоморфизмов $A_{j}$ в $A_{i}$, причем

1) $\forall i, j \forall \gamma_{1}, \gamma_{2} \in \alpha_{i j}$ ( $\gamma_{1}$ и $\gamma_{2}$ образуют регулярную пару),

2) $\forall i\left(\alpha_{i i}=G_{i}\right)$,

3) $E$ замкнуто относительно суперпозиции (пара $\left\langle\left\{A_{i}\right\}_{i \in I},\left\{\alpha_{i j}\right\}_{i, j \in I, i \leqslant} j\right\rangle$ является категорией).

ОПРЕДЕЛЕНИЕ 15. Двойным разбиением натурального ряда чисел назовем совокупность множеств $\left\{C_{j}^{i}\right\}_{i \in I, 0 \leqslant j \leqslant k_{i}}$ такую, что

1) $I$ - конечное множество индексов,

2) множества $B_{i}=\bigcup_{j} C_{j}^{i}$ являются рекурсивными для всех $i$ и образуют разбиение $\mathbb{N}$, т.е. $\bigcup_{i} B_{i}=\mathbb{N}$ и $\forall i_{1}, i_{2}\left(i_{1} \neq i_{2} \rightarrow B_{i_{1}} \cap B_{i 2}=\varnothing\right)$,

3 ) для любого $i$ множества $\left\{C_{j}^{i}\right\}_{j=0}^{k_{i}}$ образуют разбиение $B_{i}$ на $k_{i}$ (конечное число или $\left.\aleph_{0}\right)$ конечных множеств одинаковой мощности. 
ОПРеДЕЛЕниЕ 16. Скажем, что сеть вложений $\mathrm{E}=\left\langle\left\{A_{i}\right\}_{i \in I}, \leqslant{ }_{D},\left\{G_{i}\right\}_{i \in I}, E\right\rangle$ и двойное разбиение $M=\left\{C_{j}^{i}\right\}_{i \in I, 0 \leqslant j \leqslant k_{i}}$ согласованьи, если

1) $\forall i \in I\left(A_{i}=C_{0}^{i}\right)$,

2) для каждого $i$ на множестве $B_{i}=\bigcup_{j} C_{j}^{i}$ определена пара рекурсивных функций $J_{1}^{i}, J_{2}^{i}$ таких, что $\forall n<k_{i}\left(J_{1}^{i}\left(C_{n}^{i}\right)=C_{n+1}^{i}\right), J_{1}^{i} / C_{k_{i}}^{i}=\mathrm{id} / C_{k_{i}}^{i}$ (если $k_{i}$ конечно), $J_{2}^{i} / C_{n+1}^{i}=\left(J_{1}^{i}\right)^{-1}\left(C_{n}^{i}\right), J_{2} / C_{0}^{i}=\mathrm{id} / C_{0}^{i}$.

ОПРЕДЕЛЕНИЕ 17. С каждой парой $(M$, Е) согласованных $M$ и Е свяжем следуюший класс обшерекурсивных функций и предикатов $\Gamma_{(M, \mathrm{E})}$ :

$$
\begin{aligned}
& \left\{P \mid \forall x, y, i, j\left(x, y \in C_{j}^{i} \& P(x) \rightarrow P(y)\right)\right\} \\
& \cup\left\{f \mid \forall i_{1}, j_{1} \exists i_{2}, j_{2} \exists \gamma \in \alpha_{i_{1} i_{2}}\left(i_{2} \leqslant D i_{1} \& \forall x \in C_{j_{1}}^{i_{1}}\left(f(x)=\left(J_{1}^{i_{2}}\right)^{j_{2}} \gamma\left(J_{2}^{i_{1}}\right)^{j_{1}}(x)\right)\right)\right\} .
\end{aligned}
$$

ТЕОРема 18 [30]. Подалгебра алгебры $\left\langle\mathscr{F} 1, \mathrm{FDA}_{=}\right\rangle$достижима сверху за конечное число иагов тогда и только тогда, когда она представима в виде $\Gamma_{(M, \mathrm{E})}$ для некоторой согласованной пары $(M, \mathrm{E})$.

В [26] описана структура атомов алгебры $\left\langle\mathscr{F}_{0}^{1}, \mathrm{FDA}_{=}\right\rangle$, т.е. минимальных элементов в множестве подалгебр за вычетом подалгебры $U_{0}$.

TЕОРема 19. Подалгебра $\mathfrak{A}$ является атомом тогда и только тогда, когда $\mathfrak{A}=[P]$ для некоторого предиката $P(\neq$ TRUE, $\neq$ FALSE $)$ или $\mathfrak{A}=\{f\}$, где $f(\neq \mathrm{id})$ - рекурсивная перестановка $\mathbb{N}$ такая, что $\exists p$ ( $p$ - простое число $\left.\& \forall x\left(f^{p}(x)=x \& \forall i\left(1 \leqslant i \leqslant p \rightarrow f^{i}(x) \neq x\right)\right)\right)$.

Перестановки, используемые в теореме 19, назовем циклическими.

При дальнейшем проникновении вглубь структуры подалгебр трудности описания резко возрастают.

ОПРЕДЕЛЕНИЕ 18. Подалгебру $\mathfrak{A}$ назовем покрытием атома, если $\exists \mathfrak{B} \forall \mathfrak{C}$ $(\mathfrak{B}$ атом $\& \mathfrak{B} \subseteq \mathfrak{A} \&(\mathfrak{B} \subseteq \mathfrak{C} \subseteq \mathfrak{A} \rightarrow \mathfrak{B}=\mathfrak{C} \vee \mathfrak{C}=\mathfrak{A}))$

TЕОРема 20. $\mathfrak{A}$ является покрытием атома тогда и только тогда, когда

1) $\mathfrak{A}=[P, Q]$ для некоторых предикатов $P, Q(\neq$ TRUE, $\neq$ FALSE, $P \neq\rceil Q)$ или

2) $\mathfrak{A}=[P, f]$, əде $f-$ ииклическая, $P \neq$ TRUE, $\neq$ FALSE, $u \forall x(P(x) \rightarrow p(f(x)))$, или

3) $\mathfrak{A}=[P, f]$, дде $f$ - ииклическая, $P \neq$ TRUE, $\neq$ FALSE, и в каждом иикле $\left\{x, \ldots, f^{n-1}(x)\right\}$ функции $f$ предикат $P$ истинен ровно на одном әлементе, или

4) $\mathfrak{A}=[f, g]$, где $f, g$ - ииклические и группа $G$ перестановок $\mathbb{N}$, порождаемая функииями $f, g$, является полурегулярной и обладает тем свойством, что любая ее нетривиальная подгруппа, содержащая перестановку $f$, совпадает $c[f]$.

Группа $G$ в пункте 4) теоремы 20 может быть и бесконечной - группой Бернсайда. Сушествование групा Бернсайда с указанным свойством доказано А. Ольшанским [20]. 
ОПРЕДЕЛЕНИЕ 19. ПодалГебра $U$ не имеет бесконечных чепей подалгебр, если \rceil$\exists U_{1}, \ldots, U_{n}, \ldots \forall i\left(U_{i}<U_{i+1} \& U_{i}<U\right)$. Множество всех таких подалгебр обозначим $V$.

ТЕОРЕМа 21. V не является верхней полурешеткой.

Доказательство теоремы принципиально зависит от факта существования групп Ольшанского. Если бы таких групп не было, то множество $V$ оказалось бы верхней полурешеткой.

Структура подалгебр алгебры $\left\langle\mathscr{F}_{0}^{1}, \mathrm{FDA}_{=}\right\rangle$является, по сушеству, расширением множества $T$-степеней неразрешимости на субрекурсивньй случай [26]. Тем не менее имеет место следующий результат:

Теорема 22. Структура Т-степеней неразрешимости и структура подалгебр алгебры $\left\langle\mathscr{F}_{0}^{1}, \mathrm{FDA}_{=}\right\rangle$, рассматриваемые в сигнатуре $\leqslant$, не являются әлементарно әквивалентными.

Доказательство этой теоремы использует факт невложимости в множество $V$ трехэлементной решетки $\langle 0, a, 1\rangle$ с $0<a<1$ в качестве начального сегмента.

Для алгебры $\left\langle\mathscr{F}_{0}^{1}, \mathrm{FDAA}_{=}\right\rangle$структура подалгебр несколько проше, как и следовало ожидать, учитьвая, что класс FDAA = является более сильным средством замыкания. В частности, удается описать структуру всех конечно-порожденных подалгебр.

ТЕОРемА 23 [28]. $\mathfrak{A}$ является конечно-порожденной подалгеброй $\left\langle\mathscr{F}_{0}^{1}, \mathrm{FDAA}_{=}\right\rangle$ тогда и только тогда, когда $\exists n \exists f_{1}, \ldots, f_{n} \exists m \exists p_{1}, \ldots, p_{m}$

$$
\begin{aligned}
&(\mathfrak{A}=\{f \mid \forall x, y\left.\left(y=f(x) \rightarrow \exists l \exists k_{1}, \ldots, k_{l}\left(y=f_{k_{1}} \ldots f_{k_{l}}(x)\right)\right)\right\} \\
&\left.\cup\left\{p \mid p / M-\text { булева комбиначия предикатов } p_{1}, \ldots, p_{m}\right\}\right),
\end{aligned}
$$

где $M=\{x \mid \forall f \in \mathfrak{A}(f(x)=x)\}$.

Описание структуры замкнутых классов позволяет продвинуться в решении проблемы выразимости, состояшей (в рассматриваемых алгебрах) в решении вопроса: можно ли заданную функцию или предикат выразить через заданный набор функций и предикатов.

Так, из теоремы 23 непосредственно вытекает следующий критерий выразимости (для функций) в алгебре $\left\langle\mathscr{F}_{0}^{1}, \mathrm{FDAA}_{=}\right\rangle$.

СлЕДСТВИЕ. Функиия $f$ выразима через набор $\left\langle f_{1}, \ldots, f_{n}\right\rangle$ тогда и только тог$\partial a, \kappa о г \partial a$

$$
\forall x, y \quad\left(y=f(x) \leftrightarrow \exists l \exists k_{1}, \ldots, k_{l} \leqslant n\left(y=f_{k_{1}} \ldots f_{k_{l}}(x)\right)\right)
$$




\section{6. Конгруэнция и автоморфизмы}

ОПРЕДЕЛЕНИЕ 20. Изоморфизмом подалгебры $\mathfrak{A}$ на подалгебру $\mathfrak{B}$ назовем такое взаимно-однозначное отображение $\varphi \mathfrak{A}$ на $\mathfrak{B}$, которое отображает функции в функции и предикаты в предикаты и которое сохраняет операцию замыкания относительно схем программ, т.е. если схема программ $S$ при некоторой интерпретации $I$ вычисляет функцию $h$ (предикат $r$ ), то $S$ при интерпретации $\varphi(I)=\left\{\varphi\left(q_{i}\right) \mid q_{i} \in I\right\}$ вычисляет функцию $\varphi(h)$ (предикат $\varphi(r))$.

Автоморфизмом называется изоморфизм алгебры на себя.

В ряде работ изучались автоморфизмы как всей алгебры рекурсивных функций и предикатов, так и изоморфизмы между максимальными подалгебрами.

В [26] описаны все автоморфизмы алгебры $\left\langle\mathscr{F}_{0}^{1}, \mathrm{FDA}_{=}\right\rangle$.

Если $\alpha$ - рекурсивная перестановка $\mathbb{N}$, то индуцируемое ей отображение $\varphi_{\alpha}: \mathscr{F}_{0}^{1} \rightarrow \mathscr{F}_{0}^{1}$, определяемое следуюшим образом: $\varphi_{\alpha}(f)=\alpha f \alpha^{-1}$ для функции $f$, $\varphi(p)=p \alpha^{-1}$ для предиката $p$, является автоморфизмом $\left\langle\mathscr{F}_{0}^{1}, \mathrm{FDA}_{=}\right\rangle$и называется внутренним.

ТЕОрема 24. Автоморфизмы $\left\langle\mathscr{F}_{0}^{1}, \mathrm{FDA}_{=}\right\rangle$исчерпьваются внутренними.

Сформулированный результат может быть перенесен и на все остальные классы схем программ. Программные алгебры в отношении автоморфизмов также принципиально отличаются от алгебр Робинсон, у которых вообще нет нетривиальных автоморфизмов [14].

Для алгебры $\left\langle\mathscr{F}_{r}^{1}, \operatorname{FDS}(1)\right\rangle$ в [4] ряд описанных максимальных подалгебр расклассифицирован с точностью до изоморфизма. В частности, показано, что сушествует максимальная подалгебра, не изоморфная никакой другой максимальной подалгебре, а также, что в семействе максимальных подалгебр из теоремы 11 алгебры $\mathfrak{A}_{A}$ и $\mathfrak{A}_{B}$ изоморфны тогда и только тогда, когда $|A|=|B|$.

Рассматривались изоморфизмы не только отдельных алгебр, а всего множества подалгебр целиком. Множество подалгебр любой алгебры образует частично упорядоченное множество относительно отношения включения. Взаимно-однозначные отображения этого множества на себя, сохраняющие отношение включения, являются автоморфизмами.

Имеет место следующая

Tеорема 25 [26], [44]. Множсество авутоморфизмов структуры подалгебр алгебры $\left\langle\mathscr{F}_{0}^{1}, \mathrm{FDA}_{=}\right\rangle$исчерпьвается внутренними автоморфизмами.

Следует отметить, что проблема описания автоморфизмов множества $T$-степеней до сих пор остается открытой, относящейся к числу наиболее сложных проблем теории алгоритмов [40].

ОПРЕДЕЛЕНИЕ 21. Конгруәнцией на алгебре $\mathfrak{A}$ назьвается отношение эквивалентности $\sim$ на $\mathfrak{A}$ такое, что

а) в отношении может находиться пара функций либо пара предикатов, но не предикат и функция, 
б) для любой схемы программ $S$, если она в интерпретации $I$ вычисляет функцию $f$ (предикат $p$ ), то в интерпретации $I^{\prime}$, получающейся заменой функций и предикатов из $I$ на функции и предикаты, находжшиеся с ними в отношении $\sim$, схема $S$ вычисляет функцию (предикат), также находящуюся с функцией $f$ (предикатом $p$ ) в отношении $\sim$.

Алгебра назьвается простой, если она не имеет конгруэнций, отличных от тривиальных, т.е. единичной, при которой все элементы эквивалентны, и нулевой, при которой все элементы различны.

Конгруэнции на программных алгебрах рекурсивных функций изучались в рабо$\operatorname{Tax}[4],[26]$.

В [26] дано полное описание всех простых конечно-порожденных подалгебр алгебры $\left\langle\mathscr{F}_{0}^{1}, \mathrm{FDA}_{=}\right\rangle$.

ТЕОРема 26. Подалгебра $\mathfrak{A}$ является простой тогда и только тогда, когда $\mathfrak{A}=U_{0}$ или $\mathfrak{A}$ содержит функиии, отличные от id, и для любого $d$ такого, что $\exists e \neq d \exists f \in \mathfrak{A}(f(d)=e)$ и любого не тождественно истинного предиката вида $f(x)=g(x)$, где $f, g \in \mathfrak{A}$, имеет место $\exists u \exists h \in \mathfrak{A}(h(d)=u \& f(u) \neq g(u))$.

Применяя это описание, легко показать, что алгебра $\left\langle\mathscr{F}_{0}^{1}, \mathrm{FDA}=\right\rangle$ является простой, а максимальные подалгебры вида $\mathfrak{A}_{A}$ просты тогда и только тогда, когда $|A|=1$.

В [4] показано, что алгебра $\left\langle\mathscr{F}_{r}^{1}, \operatorname{FDS}(1)\right\rangle$ также является простой, а все ее подалгебры вида $\mathfrak{A}_{A}$ не просты.

\section{7. Абстрактная вычислимость в алгебраических системах}

Задачей абстрактной вычислимости согласно [11] является описание вычислимости в алгебраических системах $\langle A, \mathscr{L}\rangle$ в предположении вычислимости всех функций и предикатов, входящих в $\mathscr{L}$.

Существует несколько подходов к построению теории абстрактной выгислимости.

Один из них - его можно назвать программистским - базируется на следующем определении.

ОПРЕДЕЛЕНИЕ 22. Теорией въчислимости в алгебраической системе $\langle A, \mathscr{L}\rangle$ при условии, что $\mathscr{L}$ состоит из всюду определенных функций и предикатов, назовем замыкание $\mathscr{L}$ относительно класса $\mathrm{FDA}=$.

Впервые эта возможность была рассмотрена в [36], [45]. В этих работах предложено использовать класс схем программ FDA и доказано, что замыкание $\mathscr{L}$ относительно FDA= обладает многими свойствами множества рекурсивных (в классическом смысле) функций. Сильным аргументом в пользу выбора именно этого класса схем программ можно считать то, что он является наиболее сильным, универсальным [15], по крайней мере, среди классов схем детерминированных последовательных программ, и это позволяет получить наиболее широкую теорию вычислимости в алгебраических системах.

Таким образом, объект изучения в программных алгебрах рекурсивных функций и в теории абстрактной вычислимости фактически один и тот же - замькание относительно FDA= множества $\mathscr{L}$ функций и предикатов. 
Аппарат, развитый в теории программных алгебр, позволяет получить, в ряде случаев, полное описание вычислимости в алгебраических системах.

ТЕОРема 27 [26]. Если $\langle A, \mathscr{L}\rangle$ - конечно-порожденная алгебраическая система с разрешимой проблемой тождества слов, порождающие элементы которой включены в сигнатуру $\mathscr{L}$ в качестве констант, то теория вычислимости над $\langle A, \mathscr{L}\rangle$ изоморфна вычислимости относительно некоторой $T$-степени а.

Этот результат является подтверждением гипотезы Дж. Такера [48].

В работе [27] получено полное опсиание вычислимости в некоторых полях.

ТЕОРема 28. Пусть $\mathbb{Q}(\sqrt{2})$ - расширение поля рациональных чисел и $\mathscr{L}=$ $\left\langle+,,-,()^{-1}, \leqslant, 0,1\right\rangle$ - сигнатура упорядоченного поля, причем, чтобъ остаться в рамках всюду определенных функиий, положим $0^{-1}=0$. Тогда $[\mathscr{L}]_{\mathrm{FDA}}=$ изоморфно максимальной подалгебре алгебрьц $\left\langle\mathscr{F}_{0}, \mathrm{FDA}_{=}\right\rangle$.

TEOPEMA 29. Пусть $\mathbb{Q}(\alpha)$ - алгебраическое расширение простой степени и $\mathscr{L}=\left\langle+,,-,()^{-1}, 0,1\right\rangle$. Тогда $[\mathscr{L}]_{\mathrm{FDA}}=$ изоморфно подалгебре алгебри $\left\langle\mathscr{F}_{0}, \mathrm{FDA}=\right\rangle$, достижимой сверху за два шага, т.е. "максимальной в максимальной".

При рассмотрении вычислимости в частичных алгебраических системах (т.е. если $\mathscr{L}$ содержит не всюду определенные функции и предикаты) использованных выше классов схем последовательных программ уже не достаточно. Для адекватной работы с частичньми объектами требуются [34] специальные средства параллелизма.

В [43] введен класс схем параллельных программ.

ОПРЕДЕЛЕНИЕ 23. СХема параллельных программ с массивами и равенством $\left(\mathrm{PFDA}_{=}\right)$- это конечный ориентированньй граф, вершины которого помечены инструкциями того же вида, что и в определении 1 , а также могут быть помечены инструкцией AND.

Из вершины с инструкцией AND выходят два непомеченных ребра. Неформально семантика оператора AND такова: при его выполнении вычисления делятся на два полностью независимых и не взаимодействующих процесса вьгислений (ветви), следуя двум выходящим ребрам графа.

Программа, использующая оператор AND, будет вычислять, вообще говоря, многозначную функцию (или предикат). Запись $z \prec f\left(x_{1}, \ldots, x_{n}\right)$ означает, что $z$ является одним из значений функции $f$ на аргументах $x_{1}, \ldots, x_{n}$ (z вычисляется на одной из ветвей).

В [43] ряд результатов, полученных ранее для алгебры $\left\langle\mathscr{F}_{0}, \mathrm{FDA}_{=}\right\rangle$, перенесен на алгебру $\left\langle\mathscr{F}_{r}, \mathrm{PFDA}_{=}\right\rangle$.

Вычислимость в алгебраических системах посредством PFDA= оказалась также тесно связанной с другими концепциями вычислимости.

ОПРЕДЕЛЕНИЕ 24. Функция $f: A^{n} \rightarrow A$ называется поисково-вычислимой на алгебраической системе $\langle A, \mathscr{L}\rangle$ тогда и только тогда, когда существует рекурсивно перечислимое множество $H=\left\{\left\langle F_{i}, G_{i}\right\rangle\right\}_{i=0}^{\infty}$, где $F_{i}$ - бескванторная формула сигнатуры 
$\mathscr{L} \cup\{=\}, G_{i}-$ терм сигнатуры $\mathscr{L}$, такое, что

$$
\begin{array}{r}
f\left(x_{1}, \ldots, x_{n}\right) \succ z \leftrightarrow \exists i \exists y_{1}, \ldots, y_{p_{i}}\left(F_{i}\left(x_{1}, \ldots, x_{n}, y_{1}, \ldots, y_{p_{i}}\right)\right. \\
\left.\& G_{i}\left(x_{1}, \ldots, x_{n}, y_{1}, \ldots, y_{p_{i}}\right)=z\right) .
\end{array}
$$

Поисково-вычислимые функции были введены Я. Московакисом [41], определение приведено согласно [35].

Tеорема 30 [29]. Если $\mathscr{L}$ такова, что $\forall x, y \exists \tau(\tau-$ терм сигнатурь $\mathscr{L} \& \tau(x)=y)$, то $\forall f\left(f\right.$ поисково-вычислима $\left.\leftrightarrow f \in[\mathscr{L}]_{\mathrm{PFDA}_{=}}\right)$.

Схемы программ из класса PFDA= могут использоваться и для вычисления функционалов, т.е. отображений множества функций на $\mathbb{N}$ в себя.

ОПРЕДЕЛЕНИЕ 25. Пусть схема $S \in \mathrm{PFDA}_{=}$вычисляет некоторую (многозначную) функцию и все ее предикатные символы интерпретированы рекурсивньми предикатами, а все функциональные символы, кроме одного, $f,-$ рекурсивными функциями.

Если при подстановке вместо символа $f$ любой частичной функции $g$ (на $\mathbb{N}$ ) получившаяся в результате полностью интерпретированная схема вычисляет некоторую однозначную функцию $h$, то скажем, что схема $S$ представляет функиионал $\Psi$, отображающий $g$ и $h$.

Приведем в слегка переформулированном виде результат из [43].

Теорема 31. $h \leqslant_{e} g \leftrightarrow \exists \Psi(h=\Psi(g) \& \Psi-$ функиионал, представимый схемой из класса $\left.\mathrm{PFDA}_{=}\right)$.

\section{СПИСОК ЛИТЕРАТУРЫ}

[1] Барздинь Я.М. Об одном классе машин Тьюринга (машины Минского) // Алгебра и логика. 1962. Т. 1. С. 42-51.

[2] Глушков В.М. Теория автоматов и формальные преобразования микропрограмм // Кибернетика. 1965. № 5. С. 1-9.

[3] Глушков В. М., Цейтлин Г. Е., Ющенко Е. Л. Алгебра. Языки. Программирование. Киев: Наукова Думка, 1978.

[4] Голунков Ю. В. К теории систем алгоритмических алгебр // ДАН СССР. 1977. Т. 232. № 4. C. $749-752$.

[5] Голунков Ю. В. О полноте операций в системах алгоритмических алгебр // Алгоритмы и автоматы. Казань: КГУ, 1978. С. 11-53.

[6] Голунков Ю. В. О предполных классах алгоритмов, сохраняющих принадлежность множеству, 1 // Вероятностные методы и кибернетика. № 16. Казань: КГУ, 1980. С. 21-40.

[7] Голунков Ю.В. Аппроксимационная полнота в алгебрах частично рекурсивных функций и предикатов // Кибернетика. 1987. №6. С. 26-30.

[8] Голунков Ю.В. Степень неразрешимости проблемы полноты в алгебрах рекурсивных функций // Матем. заметки. 1988. Т. 44. № 5. С. 620-627.

[9] Голунков Ю.В. Полнота с заданной точностью в функциональных системах программного типа // Дискретн. матем. 1990. Т. 2. № 3. С. 42-49.

[10] Ершов А. П. Операторные алгоритмы, 1 // Проблемы кибернетики. № 3. М.: Физматгиз, 1960. C. $5-48$. 
[11] Ершов А. П. Вычислимость в произвольных областях и базисах // Семиотика и информатика. 1982. № 19. С. 3-53.

[12] Ершов А.П., Ляпунов А. А. О формализации понятия программы // Кибернетика. 1967. №5. С. 40-57.

[13] Заславский И. Д. Граф-схемы с памятью // Труды МИАН. 1964. Т. 72. С. 99-192.

[14] Захаров Д. А. Рекурсивные функции. Новосибирск: НГУ, 1970.

[15] Котов В.Е., Саберфельд В. К. Теория схем программ. М.: Наука, 1991.

[16] Кудрявцев В. Б. Функциональные системы. М.: МГУ, 1982.

[17] Марченков С. С. Базисы по суперпозиции в классах рекурсивных функций // Матем. вопросы кибернетики. № 3. М.: Наука, 1991. С. 115-139.

[18] Минский М. Вычисления и автоматы. М.: Мир, 1971.

[19] Непомнящий В.А.Критерий алгоритмической полноты систем операций // Теория программирования. Т. 1. Новосибирск: ВЦ СОАН СССР, 1972. С. 267-279.

[20] Ольшанский А. Ю.Группы ограниченного периода с подгруппами простого порядка // Алгебра и логика. 1982. Т. 21. № 5. С. 553-618.

[21] Роджерс Х. Теория рекурсивных функций и эффективная вычислимость. М.: Мир, 1972.

[22] Савельев А. А. Об одном способе описания максимальных подсистем алгебр // Вероятностные методы и кибернетика. № 18. Казань: КГУ, 1982. С. 77-83.

[23] Соловьев В. Д. Критерий полноты систем вычислимых функций и предикатов // V Всесоюзн. конф. по пробл. теорет. кибер. Тезисы докл. Новосибирск: ИМ СОАН СССР, 1980. C. 115 .

[24] Соловьев В. Д. Структура замкнутых классов вычислимых функций и предикатов // Изв. ВУЗов. Математика. 1982. №12. С. 51-56.

[25] Соловьев В. Д. Полнота систем общерекурсивных функций и предикатов I; II // Изв. ВУЗов. Математика. 1989. № 8; 9. С. 56-63; 60-66.

[26] Соловьев В. Д. Алгебраические аспекты абстрактной теории вычислимости // Матем. вопросы кибернетики. № 3. М.: Наука, 1991. С. 233-256.

[27] Соловьев В. Д. Вычислительные теории над алгебрами // Международн. конф. по алгебре. Новосибирск: ИМ СОАН СССР, 1991. С. 135-136.

[28] Соловьев В. Д. Программные алгебры рекурсивных функций с дополнительными средствами замькания // Изв. ВУЗов. Математика. 1993. №6. С. 46-52.

[29] Соловьев В. Д. Вычислимость недетерминированными программами и поисковая вычислимость Московакиса // Матем. заметки. 1994. Т. 56. №1. С. 149-152.

[30] Соловьев В. Д. Программно-замкнутые классы общерекурсивных функций и предикатов конечного ранга // Изв. ВУЗов. Математика. 1993. № 9. С. 51-63.

[31] Яблонский С. В. Функциональные построения в $k$-значной логике // Труды МИАН. 1958. T. 51. C. 109-140.

[32] Янов Ю.И. О логических схемах алгоритмов // Проблемы кибернетики. Т. 1. М.: Физматгиз, 1958. С. $75-127$.

[33] Aho A., Hopcroft j., Ullman J. The design and analysis of computer algorithms. London: Addison-Wesley, 1976.

[34] Constable R., Gries D. On classes of program schemata // SIAM Comput. 1972. V. 1. P. 66-108.

[35] Gordon C. E. Prime and search computability, characterized as definability in certain sublanguages of constructible L // Trans. Amer. Math. Soc. 1974. V. 197. P. 391-408.

[36] Friedman H. Algorithmic procedures, generalized Turing algorithms and elementary recursion theory // Logic Coll.'69. Amsterdam: North-Holland, 1971. P. 361-389.

[37] Kfoury A. J. Definability by programs in first-order structures // Theor. Comp. Sci. 1983. V. 25. P. 1-66.

[38] Kfoury A. J., Urzyczyn P. Necessary and sufficient conditions for the universality of programming formalisms // Acta Infor. 1985. V. 22. P. 347-377. 
[39] Kfoury A. J., Urzyczyn P. Concurrent program schemes // Fundam. Infor. 1987. V. 10. P. 337-362.

[40] Lerman M. Degrees of unsolvability. Berlin: Springer-Verlag, 1983.

[41] Moshovakis Y. N. Abstract first order computability, I; II // Trans. Amer. Math. Soc. 1969. V. 138. P. 427-504.

[42] Post E. Two-valued iterative systems of mathematical logic // Ann. Math. Studies. V. 5. Prinseton, 1941.

[43] Soloviev V.D. Nondeterministic finite algorithmic procedures as the models of abstract computability // Lect. Notes Comp. Sci. V. 278. Berlin: Springer-Verlag, 1987. P. 409-411.

[44] Soloviev V.D. Automorphism of the program closed classes of functions and predicates structure // Recursive Function Theory: Newsletter. 1992. №39.

[45] Spepherdson L.S. Computation over abstract structures: serial and parallel procedures and Friedman's definitional schemes // Logic Coll.'73. Amsterdam: North-Holland, 1975. P. 445-513.

[46] Sheperdson J. C., Sturgis H. E. Computability of recursive functions // Jour. ACM. 1963. V. 10. № 2. P. 217-255.

[47] Tokura N., Kasami T., Furuta Sh. Janov schemas augmented by a pushdown memory // IEEE 15th Ann. Symp. Switch and Automata theory. New York, 1974. P. 84-94.

[48] Tucker J. V. Computing in algebraic systems // Preprint series. Oslo: Matem. Inst. Univ. i Oslo, 1978. 